UT-920

hep-th/0012254

December, 2000

\title{
A D2-brane realization of Maxwell-Chern-Simons-Higgs systems
}

\author{
Yosuke Imamura* \\ Department of Physics, University of Tokyo, Tokyo 113-0033, Japan
}

\begin{abstract}
We show that $\mathcal{N}=2$ supersymmetric Maxwell-Chern-SimonsHiggs systems in three dimension can be realized as gauge theories on a D2-brane in D8-branes background with a non-zero $B$-field. We reproduce a potential of Coulomb branch of the Chern-Simons theory as a potential of a D2-brane in a classical D8-brane solution and show that each Coulomb vacuum is realized by a D2-brane stabilized in the bulk at a certain distance from D8-branes.
\end{abstract}

*imamura@hep-th.phys.s.u-tokyo.ac.jp 


\section{Introduction}

Chern-Simons theories in three-dimension [1] have many interesting properties: rich phase structures, existence of solitons, etc. In this paper, we discuss their brane realization.

One way to realize three-dimensional gauge theories in string theory is to use Hanany-Witten type configurations. $\mathcal{N}=4$ gauge theories are realized on D3-branes stretched between parallel NS5-branes [2]. We can decrease the number of supersymmetries and can introduce a non-zero Chern-Simons coupling by replacing NS5-branes by $(p, q)$ 5-branes and putting them at appropriate angles [3, 4, 5, 6, 7, 8]. Using these configurations, moduli space, phase structures and solitons of the theories are studied in geometrical ways.

We can also realize three-dimensional gauge theories as theories on D2-branes [9], 10]. For example, an $\mathcal{N}=4 U(1)$ gauge theory with $N_{f}$ flavors is realized on a D2-brane in $N_{f}$ D6-branes background. However, unlike the Hanany-Witten type configurations, we come up against a problem if we attempt to introduce a Chern-Simons term. The D2-brane action involves the following term[11]

$$
S=\frac{\Lambda}{4 \pi} \int A \wedge d A
$$

where $\Lambda$ is a cosmological constant quantized in integer units. Because we need to use this coupling to introduce a nonzero Chern-Simons coupling, we should put D2-branes in a massive IIA or, equivalently, a D8-brane background. If we put a D2-brane parallel to a D8-brane, however, the configuration becomes non-BPS and unstable. In order to keep supersymmetries unbroken, we have to take one direction on the D2-brane to be a dilatonic direction perpendicular to the D8-brane. Although $\mathcal{N}=(8,0)$ supersymmetry is realized in this case, the three-dimensional Poincaré invariance is broken 12.

In [13] open string spectra and BPS conditions of $\mathrm{D} p-\mathrm{D} p p^{\prime}$ systems are studied and it is pointed out that $\mathrm{D} p-\mathrm{D}(p+6)$ systems can be made BPS by turning on a constant $B$-field. Recently, Mihailescu, Park and Tran 14 and Witten 15 take advantage of this fact to study gauge theories on D-branes. In this paper, inspired by their idea, we study a relation between a D2-D8 configuration with a non-zero $B$-field and a Maxwell-Chern-Simons-Higgs system expected to be realized on the D2-brane.

\section{Maxwell-Chern-Simons-Higgs systems}

We consider a D2-D8 system with a non-zero $B$-field. Let us assume that $N_{f}$ D8-branes are located at $x^{9}=q_{I}\left(I=1,2, \ldots, N_{f}\right)$ and one D2-brane spreads along the $x^{0}, x^{1}$ and $x^{2}$ directions. We turn on the following components of the 
$B$-field.

$$
\frac{B_{36}}{T}=\tan \theta_{1}, \quad \frac{B_{47}}{T}=\tan \theta_{2}, \quad \frac{B_{58}}{T}=\tan \theta_{3}, \quad\left(0 \leq \theta_{i} \leq \pi\right),
$$

where $T=1 /\left(2 \pi l_{s}^{2}\right)$ is the string tension. This configuration is T-dual to a D0-D6 system in [13, 15] and a D3-D9 system in [14]. Therefore, we can divert results there to analysis of our D2-D8 system. A BPS condition for this system is

$$
e^{i\left(\theta_{1}+\theta_{2}+\theta_{3}\right)}=-i \text {. }
$$

We define a parameter $r$ by

$$
r=\theta_{1}+\theta_{2}+\theta_{3}-\frac{3 \pi}{2} .
$$

This parameter corresponds to the $r$ in [15]. We have fixed some ambiguities for definitions of parameters differently from [15] for later convenience. Near the BPS point $r=0$, an $\mathcal{N}=2 U(1)$ gauge theory is expected to be realized on the D2brane. The field content of this theory is one $U(1)$ vector multiplet $\left(a, A_{\mu}, \lambda, D\right)$ from 2-2 strings and $N_{f}$ chiral multiplets $\left(\phi_{I}, \psi_{I}, F_{I}\right)\left(I=1,2, \ldots, N_{f}\right)$ from 2-8 strings. Precisely, there are three more chiral multiplets from 2-2 strings representing fluctuations of the D2-brane parallel to the D8-branes. Because they are neutral under the $U(1)$ and decoupled from other fields, we shall neglect them in what follows.

Let us consider an $\mathcal{N}=2$ supersymmetric action for these fields. Kinetic terms of vector and chiral multiplets are

$$
\begin{aligned}
\mathcal{L}_{\text {vector-kin }} & =\frac{1}{g^{2}}\left(-\frac{1}{4} F_{\mu \nu} F^{\mu \nu}-\frac{1}{2} \partial_{\mu} a \partial^{\mu} a+\frac{1}{2} D^{2}\right), \\
\mathcal{L}_{\text {chiral-kin }} & =\frac{1}{g^{2}} \sum_{I=1}^{N_{f}}\left(-\mathcal{D}_{\mu} \bar{\phi}_{I} \mathcal{D}^{\mu} \phi_{I}+\bar{F}_{I} F_{I}+D\left|\phi_{I}\right|^{2}+a \bar{F}_{I} \phi_{I}+a \bar{\phi}_{I} F_{I}\right) .
\end{aligned}
$$

(We are now interested in only bosonic fields.) For chiral multiplets, we can add mass terms

$$
\mathcal{L}_{\text {chiral-mass }}=-\frac{1}{g^{2}} \sum_{I=1}^{N_{f}} M_{I}\left(\phi_{I} \bar{F}_{I}+\bar{\phi}_{I} F_{I}\right) .
$$

Because gauge group is $U(1)$, we can introduce a Fayet-Iliopoulous term

$$
\mathcal{L}_{\mathrm{FI}}=\frac{\xi}{g^{2}} D
$$

Furthermore, in three-dimension, a Chern-Simons term is allowed.

$$
\mathcal{L}_{\mathrm{CS}}=\frac{k}{4 \pi} \epsilon^{\alpha \beta \gamma} A_{\alpha} \partial_{\beta} A_{\gamma},
$$


where $k$ is an integer-valued quantized Chern-Simons coupling. The equation of motion for the gauge field is

$$
\partial_{\mu} F^{\mu \lambda}+\frac{\mu}{2} \epsilon^{\lambda \mu \nu} F_{\mu \nu}=\text { current, } \quad\left(\mu=\frac{k g^{2}}{2 \pi}\right) .
$$

Because the second term on the left hand side plays a role of a 'mass term' of the gauge field, we should introduce mass terms also for other component fields in the vector multiplet in order to make the action supersymmetric. [16, 17]

$$
\mathcal{L}_{\text {vector-mass }}=-\frac{\mu}{g^{2}} a D
$$

Gathering all and eliminating auxiliary fields $F_{I}$ and $D$, we obtain

$$
V\left(a, \phi_{I}\right)=\frac{1}{g^{2}}\left[\frac{1}{2}\left(\sum_{I=1}^{N_{f}}\left|\phi_{I}\right|^{2}-\mu a+\xi\right)^{2}+\sum_{I=1}^{N_{f}}\left(a-M_{I}\right)^{2}\left|\phi_{I}\right|^{2}\right] .
$$

This is a classical potential. Due to a quantum effect, the Chern-Simons coupling $k$ get a correction. Because $k$ is quantized in integer units, it cannot vary continuously. By calculating a one loop diagram of fermion fields $\psi_{I}$, we find the coupling $k(a)$ jumps by one at $a=M_{I}$ for each $I[18]$. Therefore, $\xi-\mu a$ in the classical potential (12) should be replaced by a function $h(a)$ satisfying

$$
\frac{d}{d a} h(a)=-\frac{g^{2}}{2 \pi} k(a)
$$

Finally, we obtain a quantum potential for this theory.

$$
V\left(a, \phi_{I}\right)=\frac{1}{g^{2}}\left[\frac{1}{2}\left(\sum_{I=1}^{N_{f}}\left|\phi_{I}\right|^{2}+h(a)\right)^{2}+\sum_{I=1}^{N_{f}}\left(a-M_{I}\right)^{2}\left|\phi_{I}\right|^{2}\right] .
$$

In what follows, we shall use a parameter $\xi$ as what represents a height of a 'plateau' of the function $h(a)$. (Fig.1) (It is not necessary for the function $h(a)$ to have a plateau. It can be monotonically increasing or monotonically decreasing. We assume its existence just for convenience in explanations.)

Next, let us consider vacua of this theory. There are two kinds of vacua: 'Higgs vacua' and 'Coulomb vacua'.

Higgs vacua An expansion of the potential (14) in $\phi_{I}$ is

$$
V\left(a, \phi_{I}\right)=\frac{h^{2}(a)}{2 g^{2}}+\frac{1}{g^{2}} \sum_{I=1}^{N_{f}}\left(h(a)+\left(a-M_{I}\right)^{2}\right)\left|\phi_{I}\right|^{2}+\mathcal{O}\left(\phi_{I}^{4}\right) .
$$

Roughly speaking, $\phi_{I}$ is tachyonic around $a=M_{I}$ if $h\left(M_{I}\right)$ is negative. At $a=$ $M_{I}$, a potential for $\phi_{I}$ is $V=\left(1 / 2 g^{2}\right)\left(\left|\phi_{I}\right|^{2}+h(a)\right)^{2}$ and there is supersymmetric 


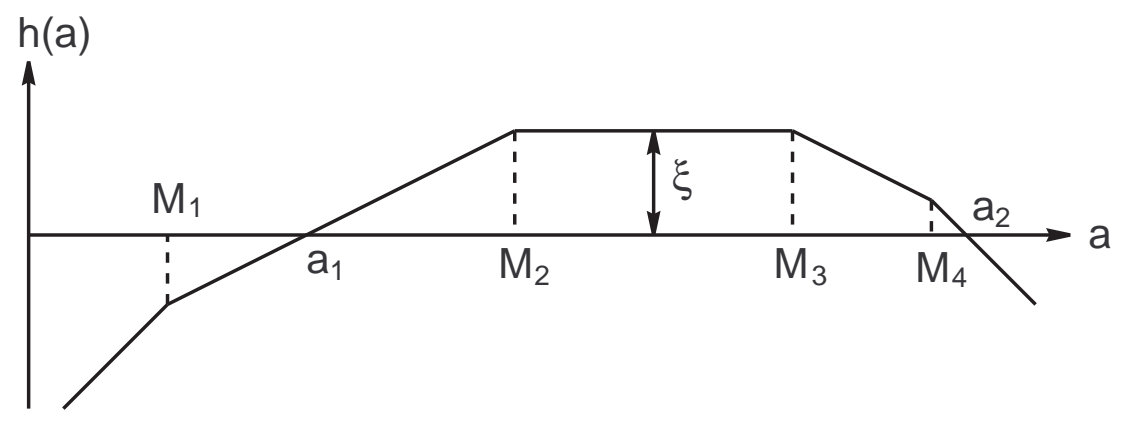

Figure 1: An example of the function $h(a)$. We define $\xi$ as a height of a 'plateau' of the function $h(a)$. In this example, there are one Higgs vacuum $\left(a=M_{1},\left|\phi_{1}\right|=\right.$ $\left.\sqrt{-h\left(M_{1}\right)}\right)$ and two Coulomb vacua $\left(a=a_{1}, \phi_{I}=0\right)$ and $\left(a=a_{2}, \phi_{I}=0\right)$.

vacuum $\left|\phi_{I}\right|=\sqrt{-h\left(M_{I}\right)}$ if $h\left(M_{I}\right)<0$. This vacuum breaks the gauge symmetry. As is mentioned in [15] for D0-D6 systems, Higgs vacuum with $\phi_{I} \neq 0$ is regarded as a true bound state of the D2-brane and the $I$-th D8-brane. Although it would be interesting problem how to realize this bound state as a classical supergravity solution, we will not argue this in this paper.

Coulomb vacua In the Coulomb branch $\phi_{I}=0$, the potential is

$$
V(a)=\frac{h^{2}(a)}{2 g^{2}} .
$$

There are three phases. If $\xi<0$, the potential $V(a)$ is everywhere positive. In this case there are supersymmetric Higgs vacua as we mentioned above and a state on the Coulomb branch decays into one of the Higgs vacua. If $\xi=0$, we have a continuous set of vacua on the plateau of the function $h(a)$. All these vacua are supersymmetric and have an unbroken gauge symmetry. For $\xi>0$, we have supersymmetric Coulomb vacua at $a$ satisfying $h(a)=0$. On these vacua, both the supersymmetry and the gauge symmetry are unbroken. The fact that only specific values of scalar field $a$ are chosen suggests an interesting phenomenon in string theory. It implies that the D2-brane is stabilized in the bulk at a certain distance from the D8-branes. In the next section, we show that the potential $V(a)$ is reproduced as one for a D2-brane and the stabilization actually takes place.

\section{A classical D8-brane solution with $B$-field}

In this section, we construct a classical solution for D8-branes in $B \neq 0$ and reproduce the potential (16) as a potential for a D2-brane in the D8-brane back- 
ground.

We can make a D8-brane solution with a non-zero $B$-field by T-dualizing a smeared D5-brane solution. Let us begin with the following D5-brane solution.

$$
\begin{aligned}
d s^{2} & =H^{-1 / 2} \eta_{\mu \nu} d x^{\mu} d x^{\nu}+H^{1 / 2} \delta_{i j} d x^{i} d x^{j}, \\
e^{\Phi} & =g_{\mathrm{str}} H^{-1 / 2}, \\
C_{012345} & =\frac{2 \pi}{g_{\mathrm{str}}}\left(2 \pi l_{s}\right)^{-6} H^{-1},
\end{aligned}
$$

where $\mu, \nu=0,1, \ldots, 5$ and $i, j=6,7,8,9$. The harmonic function $H$ satisfies the following Laplace equation in the transverse directions.

$$
\Delta_{4} H=-\left(2 \pi l_{s}\right)^{2} g_{\mathrm{str}} \rho^{(4)},
$$

where $\rho^{(4)}$ is a D5-brane density which is now taken to be

$$
\rho^{(4)}=\frac{1}{\left(2 \pi l_{s}\right)^{3}\left(-\prod_{a=1}^{3} \cos \theta_{a}\right)} \sum_{I} \delta\left(x^{9}-q_{I}\right) .
$$

The $1 / \prod \cos \theta_{a}$ factor is necessary to compensate a change of the brane density due to the rotation which we will do next. (We assume that one of $\theta_{a}$ is larger than $\pi / 2$ and other two are smaller than $\pi / 2$. Therefore this density is positive.) By integrating (20) once, we obtain

$$
\frac{d}{d x^{9}} H\left(x^{9}\right)=-\frac{g_{\text {str }}}{2 \pi l_{s}\left(-\prod_{a=1}^{3} \cos \theta_{a}\right)} \Lambda\left(x^{9}\right),
$$

where $\Lambda\left(x^{9}\right)$ is a function representing the quantized cosmological constant after the T-duality transformation. Because $\Lambda\left(x^{9}\right)$ jumps by one as $x^{9}$ crosses the position of each D8-brane and is identified with the Chern-Simons coupling $k(a)$, the function $H\left(x^{9}\right)$ has a similar form to $h(a)$. (Fig.21) These two functions, however, are not proportional to each other. $H\left(x^{9}\right)$ must not be negative while $h(a)$ may. Let us define $g_{\mathrm{str}}$ as an expectation value of $e^{\Phi}$ on the plateau of the function $H\left(x^{9}\right)$. Then, the constant part of $H\left(x^{9}\right)$ is fixed by (18), such that $H\left(x^{9}\right)=1$ on the plateau.

Rotating this solution on 3-6, 4-7, and 5-8 planes by angles $\theta_{1}, \theta_{2}$ and $\theta_{3}$, respectively, we obtain the following metric and the R-R 6 -form potential.

$$
\begin{aligned}
d s^{2} & =H^{-1 / 2}\left(-\left(d x^{0}\right)^{2}+\left(d x^{1}\right)^{2}+\left(d x^{2}\right)^{2}\right)+H^{1 / 2}\left(d x^{9}\right)^{2} \\
& +\sum_{a=1}^{3}\left(H^{-1 / 2} \cos ^{2} \theta_{a}+H^{1 / 2} \sin ^{2} \theta_{a}\right)\left(d x^{a+2}\right)^{2} \\
& +\sum_{a=1}^{3}\left(H^{-1 / 2} \sin ^{2} \theta_{a}+H^{1 / 2} \cos ^{2} \theta_{a}\right)\left(d x^{a+5}\right)^{2}
\end{aligned}
$$




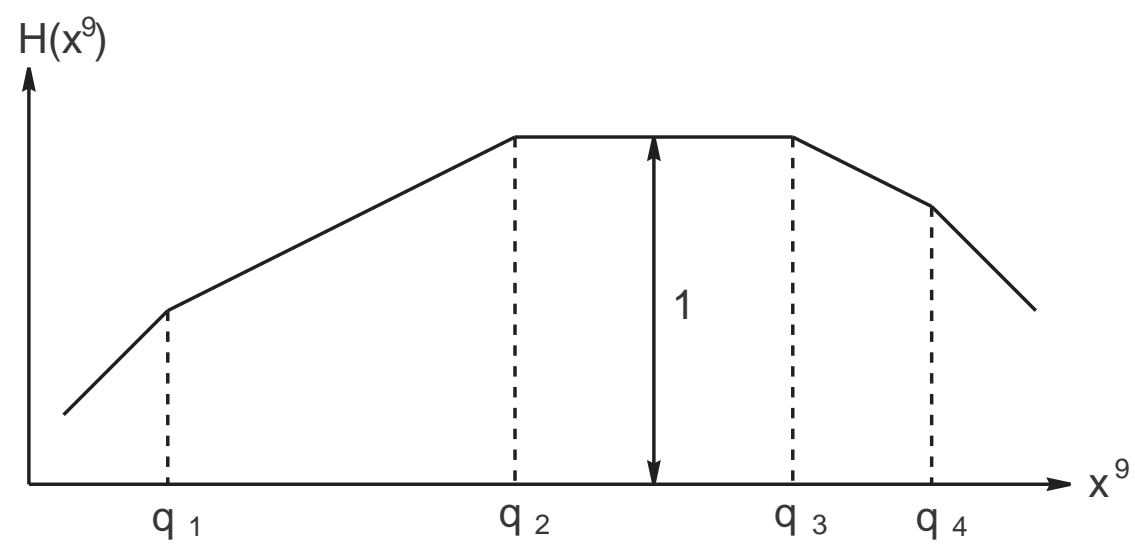

Figure 2: The harmonic function $H\left(x^{9}\right)$. Each D8-brane is located at $x^{9}=q_{I}$.

$$
\begin{aligned}
& +2 \sum_{a=1}^{3} \cos \theta_{a} \sin \theta_{a}\left(H^{-1 / 2}-H^{1 / 2}\right) d x^{a+2} d x^{a+5}, \\
C_{012678} & =\frac{2 \pi}{g_{\mathrm{str}}}\left(2 \pi l_{s}\right)^{-6} H^{-1} \sin \theta_{1} \sin \theta_{2} \sin \theta_{3} . \\
C_{012345} & =\frac{2 \pi}{g_{\mathrm{str}}}\left(2 \pi l_{s}\right)^{-6} H^{-1} \cos \theta_{1} \cos \theta_{2} \cos \theta_{3} .
\end{aligned}
$$

Other components of the R-R 6-form potential are irrelevant to our arguments.

Compactifying the $x^{6}, x^{7}$ and $x^{8}$ directions on a rectangular $\mathbf{T}^{3}$ with all period $2 \pi l_{s}$ and carrying out $\mathrm{T}$-duality transformation by relations

$$
\widetilde{G}_{a+2, a+2}=G_{a+2, a+2}-\frac{G_{a+2, a+5}^{2}}{G_{a+5, a+5}}, \quad \widetilde{G}_{a+5, a+5}=\frac{1}{G_{a+5, a+5}}, \quad(a=1,2,3),
$$

we obtain the following metric for a D8-brane solution with a nonzero $B$-field.

$$
\widetilde{G}_{\mu \nu}=H^{-1 / 2} \eta_{\mu \nu} \quad(\mu, \nu=0,1,2), \quad \widetilde{G}_{a+2, a+2}=\widetilde{G}_{a+5, a+5}=\frac{H^{1 / 2}}{F_{a}}, \quad \widetilde{G}_{99}=H^{1 / 2},
$$

where the function $F_{a}$ is defined by

$$
F_{a}=\sin ^{2} \theta_{a}+H \cos ^{2} \theta_{a}
$$

The dual dilaton field is

$$
e^{\widetilde{\Phi}}=e^{\Phi} G_{66}^{-1 / 2} G_{77}^{-1 / 2} G_{88}^{-1 / 2}=g_{\mathrm{str}} H^{1 / 4} F_{1}^{-1 / 2} F_{2}^{-1 / 2} F_{3}^{-1 / 2} .
$$

From (24), we obtain a component of the R-R 3-form potential coupling to the D2-brane as

$$
\widetilde{C}_{012}=T_{D 2} H^{-1} \sin \theta_{1} \sin \theta_{2} \sin \theta_{3} .
$$


where $T_{D 2}=1 /\left\{(2 \pi)^{2} l_{s}^{3} g_{\mathrm{str}}\right\}$ is the D2-brane tension on the plateau $H\left(x^{9}\right)=1$. The zero-form R-R field strength $\widetilde{G}$ is T-dual to $G_{678}$, which is the field strength of R-R two-form field dual to (25).

$$
\widetilde{G}=\left(2 \pi l_{s}\right)^{3} G_{678}=\frac{2 \pi}{g_{\text {str }}}\left(2 \pi l_{s}\right) H^{\prime} \cos \theta_{1} \cos \theta_{2} \cos \theta_{3}=2 \pi \Lambda .
$$

$\Lambda$ is certainly identified with the cosmological constant $\widetilde{G} / 2 \pi$ quantized in integer units as we mentioned above. Using the solution we have obtained, a potential for a D2-brane in this background is

$$
\begin{aligned}
V & =\frac{1}{(2 \pi)^{2} l_{s}^{3} e^{\widetilde{\Phi}}} \sqrt{-\widetilde{G}_{00} \widetilde{G}_{11} \widetilde{G}_{22}}-\widetilde{C}_{012} \\
& =T_{D 2} \frac{1}{H}\left[\prod_{a=1}^{3}\left(\sin ^{2} \theta_{a}+H \cos ^{2} \theta_{a}\right)^{1 / 2}-\prod_{a=1}^{3} \sin \theta_{a}\right]
\end{aligned}
$$

The following relations hold between parameters in the gauge theory and ones in string theory.

$$
k=\Lambda, \quad a=T x^{9}, \quad \frac{1}{g^{2}}=\frac{T_{D 2}}{T^{2}} .
$$

Comparing (13) and (22), we obtain the following relation between $h(a)$ and $H\left(x^{9}\right)$.

$$
h(a)-\xi=\left(-\prod_{a=1}^{3} \cos \theta_{a}\right) T(H(a / T)-1) .
$$

In order to take the field theory limit, let us expand the potential (32) around $H=1$.

$$
\frac{V}{T_{D 2}}=A\left(\theta_{a}\right)+B\left(\theta_{a}\right) \epsilon+C\left(\theta_{a}\right) \epsilon^{2}+\mathcal{O} \epsilon^{3}
$$

where $\epsilon=1-H$. Near the BPS point $r=0$, the coefficients $B\left(\theta_{a}\right)$ and $C\left(\theta_{a}\right)$ are expanded in $r$ as

$$
B\left(\theta_{a}\right)=r \cos \theta_{1} \cos \theta_{2} \cos \theta_{3}+\mathcal{O}\left(r^{2}\right), \quad C\left(\theta_{a}\right)=\frac{1}{2} \cos ^{2} \theta_{1} \cos ^{2} \theta_{2} \cos ^{2} \theta_{3}+\mathcal{O}(r) .
$$

Therefore, the potential is rewritten as

$$
V=\frac{T_{D 2}}{2 T^{2}}\left(T r+T \epsilon \cos \theta_{1} \cos \theta_{2} \cos \theta_{3}\right)^{2},
$$

up to a constant. Using (33) and (34), we can rewrite this potential in terms of variables in the field theory except the parameter $r$.

$$
V=\frac{1}{2 g^{2}}(T r+\xi-h(a))^{2},
$$


This coincides with the potential (16) if $r$ relates to the FI-parameter $\xi$ by

$$
\xi=-T r
$$

Now we have shown that the potential in the Coulomb branch of MaxwellChern-Simons-Higgs system is reproduced as a potential for a D2-brane on the D8-brane background. When $\xi>0$, which corresponds to $r<0$, we have Coulomb vacua at points with $h(a)=0$. Each of these vacua is realized by a D2-brane stabilized in the bulk at a minimum of the potential. Note that such a stabilization is impossible in case with a vanishing $B$-field. This can be seen by putting $\theta_{a}$ to be zero in (32). In this case, $V \propto H^{1 / 2}$ and there is no stable point.

\section{Discussions}

In this paper, we showed that Maxwell-Chern-Simons-Higgs systems are realized as gauge theories on a D2-brane in a D8-brane background with a nonzero $B$-field. The potential of Coulomb branch is reproduced as a potential for a D2-brane.

There are some open questions. We argued only the Coulomb branch in a supergravity framework. How can we treat the Higgs branch? One way to do it is to use a noncommutative gauge theory on D8-branes. In the decoupling limit, $r=\sum_{a}\left(\theta_{a}-\pi / 2\right)$ is proportional to $T^{-1}$. Let us take a limit in which each $\theta_{a}-\pi / 2$ is proportional to $T^{-1}$. From (2), we obtain $B_{36}, B_{47}, B_{58} \propto T^{2}$ and this is what we take in order to realize a noncommutative gauge theory on the D8-branes 19]. Higgs vacua are expected to be realized as noncommutative solitons on the D8-branes. By noncommutative parameters $\theta_{i j}$, the FI parameter $\xi$ is represented as

$$
-\xi=\frac{T^{2}}{B_{36}}+\frac{T^{2}}{B_{47}}+\frac{T^{2}}{B_{58}}=\frac{1}{\theta_{36}}+\frac{1}{\theta_{47}}+\frac{1}{\theta_{58}} .
$$

Although Higgs vacua are always supersymmetric, noncommutative soliton solutions are BPS only when $\xi=0[14$, 15]. These do not contradict to each other because in the limit $\theta_{a}-\pi / 2 \propto T^{-1}$ the expansions (36) are valid only when $\xi=r=0$. For non-zero $\xi$, it is not clear whether we can use solitonic solutions on the D8-branes for the purpose of analysis of gauge theories on the D2-branes.

A problem on supersymmetry exists for the Coulomb branch, too. For $\xi>0$, there are supersymmetric Coulomb vacua which are realized by stabilized D2branes. The D2-D8 systems, however, do not have any explicit unbroken supersymmetry.

\section{Acknowledgment}

The author would like to thank K. Ohta. Discussions with him at Summer Institute 2000 at Yamanashi, Japan was very helpful for this work. 


\section{References}

[1] G. Dunne, "Aspects of Chern-Simons theory", hep-th/9902115.

[2] A. Hanany and E. Witten, "Type IIB Superstrings, BPS Monopoles, And Three-Dimensional Gauge Dynamics",

Nucl.Phys. B492 (1997) 152, hep-th/9611230.

[3] T. Kitao, K. Ohta and N. Ohta, "Three-Dimensional Gauge Dynamics from Brane Configurations with (p,q)-Fivebrane",

Nucl.Phys. B539 (1999) 79, hep-th/9808111,

[4] K. Ohta, "Moduli Space of Vacua of Supersymmetric Chern-Simons Theories and Type IIB Branes",

JHEP 9906 (1999) 025, hep-th/9904118.

[5] B.-H. Lee, H.-j. Lee, N. Ohta and H. S. Yang, "Maxwell Chern-Simons Solitons from Type IIB String Theory", Phys.Rev. D60 (1999) 106003, hep-th/9904181.

[6] T. Kitao and N. Ohta, "Spectrum of Maxwell-Chern-Simons Theory Realized on Type IIB Brane", Nucl.Phys. B578 (2000) 215, hep-th/9908006.

[7] O. Bergman, A. Hanany, A. Karch, B. Kol, "Branes and Supersymmetry Breaking in Three Dimensional Gauge Theories",

JHEP 9910 (1999) 036, hep-th/9908075.

[8] K. Ohta, "Supersymmetric Index and s-rule for Type IIB Branes", JHEP 9910 (1999) 006, hep-th/9908120.

[9] N.Seiberg, "IR Dynamics On Branes And Space-Time Geometry", Phys.Lett.B384(1996)81, hep-th/9606017.

[10] N.Seiberg and E.Witten, "Gauge Dynamics and Compactification to Three Dimensions", hep-th/9607163. 
[11] E. Bergshoeff and P. K. Townsend, "Super D-branes",

Nucl. Phys. B490 (1997) 145, hep-th/9611173.

[12] D. Kabat and S.-J. Rey, "Wilson Lines and T-Duality in Heterotic M(atrix) Theory", Nucl. Phys. B508 (1997) 535, hep-th/9707099.

[13] B. Chen, H. Itoyama, T. Matsuo, K. Murakami " $p$-p' System with B-field, Branes at Angles and Noncommutative Geometry",

Nucl.Phys. B576 (2000) 177, hep-th/9910263.

[14] M. Mihailescu, I. Y. Park and T. A. Tran, "D-branes as Solitons of an N=1, D=10 Non-commutative Gauge Theory", hep-th/0011079.

[15] E. Witten, "BPS Bound States Of D0-D6 And D0-D8 Systems In A B-Field", hep-th/0012054.

[16] C. Lee, K. Lee and E. Weinberg, "Supersymmetry and self-dual Chern-Simons-Higgs systems", Phys. Lett. B243 (1990) 105.

[17] E. A. Ivanov, "Chern-Simons matter systems with manifest $N=2$ supersymmetry", Phys. Lett. B268 (1991) 203.

[18] O. Aharony, A. Hanany, K. Intriligator, N. Seiberg and M. J. Strassler, "Aspects of N=2 Supersymmetric Gauge Theories in Three Dimensions", Nucl. Phys. B499 (1997) 67, hep-th/9703110.

[19] N. Seiberg and E. Witten, "String Theory and Noncommutative Geometry", JHEP 9909 (1999) 032, hep-th/9908142. 